\title{
Shear Bond Strength of Brackets Bonded with Self-Etching Primers Compared to Conventional Acid-Etch Technique: A Randomized Clinical Trial
}

\section{Nasrin Farhadian, Amirfarhang Miresmaeili, Vahid Shahidi Zandi*}

Department of Orthodontics, Dental Faculty, Hamedan University of Medical Sciences, Hamadan, Iran

\begin{abstract}
Article Info
\end{abstract}
Article type:

Original Article

\section{Article History:}

Received: 6 April 2018

Accepted: 29 July 2018

Published: 30 August 2019

\section{* Corresponding author:}

Department of Orthodontics, Dental Faculty, Hamedan University of Medical

Sciences, Hamadan, Iran

Email: vahidshahidi13@gmail.com

\author{
A B S T R A C T
}

Objectives: The purpose of this randomized clinical trial (RCT) was to compare the shear bond strength (SBS) of orthodontic brackets bonded to enamel with conventional acid-etch (AE) technique and self-etching primers (SEP).

Materials and Methods: Twenty-two patients, requiring extraction of two bicuspids for orthodontic reasons, were recruited. In each individual, following blinding and allocation concealment, one intact premolar received conventional $\mathrm{AE}$, whereas the contralateral premolar received SEP with a split-mouth design. Bonded brackets remained in the oral cavity for two months. Afterward, the teeth were extracted without debonding the brackets. SBS and adhesive remnant index (ARI) were measured using a Universal Instron machine and a stereomicroscope, respectively.

Results: The mean SBS of the conventional AE and SEP groups was 9.53 and 9.20 $\mathrm{MPa}$, respectively. Paired t-test showed no statistically significant difference between the two groups $(\mathrm{P}=0.096)$. Comparison of ARI between the two groups, using Wilcoxon test, indicated that significantly less adhesive remained on enamel with brackets bonded with SEP compared to brackets bonded with conventional AE $(\mathrm{P}<0.001)$ although the SBS was higher in the AE group.

Conclusion: The present study indicated that although there is no significant difference in SBS between SEP and conventional AE for bonding orthodontic metal brackets, the amount of residual adhesive on the enamel surface is significantly less with SEP than with conventional AE.

(IRCT registration number: IRCT201705099086N3).

Keywords: Dental Bonding; Self-Etching Primer; Orthodontic Brackets; Dental Acid Etching

$>$ Cite this article as: Farhadian N, Miresmaeili A, Shahidi Zandi V. Shear Bond Strength of Brackets Bonded with Self-Etching Primers Compared to Conventional Acid-Etch Technique: A Randomized Clinical Trial. Front Dent. 2019;16(4):248-255. doi:

\section{INTRODUCTION}

A successful fixed orthodontic treatment relies on, to a large extent, preserving the adhesion between the bracket and the enamel surface. The process of etching the enamel surface was introduced by Buonocore in 1955 [1] and was employed for orthodontic attachments in 1965 by Newman [2], but it was not accepted worldwide by orthodontists until the publication of a comprehensive paper on

This work is published as an open access article distributed under the terms of the Creative Commons Attribution 4.0 License (http://creativecommons.org/licenses/by-nc/4). Non-commercial uses of the work are permitted, provided the original work is properly cited. 
direct bonding in fixed orthodontic treatment [3]. In conventional acid-etch (AE) technique, three components are used: etchant, primer, and adhesive. The most widely accepted etchant is $37 \%$ phosphoric acid. The etching process consists of applying this etchant on the enamel surface, thorough rinsing with abundant water, and drying until the observation of a frosty appearance. Next, a hydrophilic primer is applied as an intermediate layer. Afterward, a composite resin is applied [4]. The setting procedure is done with either light-curing or chemical curing [5]. It is obvious that this process is time-consuming and consists of three separate steps, leading to an increase in the chance of saliva contamination and technical errors.

To simplify the technique and shorten this process, great efforts have been made to find newer systems. Self-etching primer (SEP), introduced in 1988 by Bishara et al [6], is one of the modern bonding systems. A recent survey showed that approximately $40 \%$ of American orthodontists use this method of bonding [7]. The active ingredient of SEP is methacrylated phosphoric acid ester in which phosphoric acid and methacrylate are combined in a molecule that simultaneously etches and primes [8]. The most commonly used SEP is Transbond Plus (3M Unitek, Monrovia, CA, USA). The results of a metaanalysis indicate that Transbond Plus SEP saves about 8 minutes for full-mouth bonding compared to conventional AE [9]. Shortened time and fewer steps required for bonding contribute to reducing the chance of operator errors and the risk of blood or saliva contamination, which are the main reasons for bond failure $[10,11]$. Also, SEP has better performance in wet conditions [12].

Achieving a low rate of bond failure is a highpriority goal in orthodontics because replacement of debonded brackets is timeconsuming and costly. Direct bonding is obtained by micromechanical adhesion of the resin to etched enamel.

Bonding materials must penetrate enamel porosities. They also must have adequate shear bond strength (SBS) to preclude the bond failure resulting from masticatory and orthodontic forces [13]. On the other hand, SBS must not be so high as to cause enamel damage during debonding [12]. In-vitro studies evaluating the SBS in conventional AE and SEP groups have reached contradictory results; some studies found no significant differences [14-16], whereas the others mentioned that the SBS of the conventional AE group is significantly higher than that of the SEP group [17-19]. On the contrary, some researchers stated that the SBS of SEP is significantly higher than that of the conventional AE group $[11,12,20]$. The primary aim of this study was to compare the SBS of brackets bonded with conventional AE and SEP in a clinical situation. The null hypothesis was that there is no significant difference in the SBS of these two groups. The secondary aim was to compare the adhesive remnant index (ARI) between the two groups after bracket debonding.

\section{MATERIALS AND METHODS}

This in-vivo study with a split-mouth design was approved by the Ethics Committee of Hamadan University of Medical Sciences (code: IR.UMSHA.REC.1396.166). After the complete description of the interventions, informed consent was signed by all participants. The sample size was calculated according to a study by Mirzakouchaki et al [21], considering the study power of $80 \%$ and a significance level of $\alpha<0.05$, using the below formula:

$$
\begin{aligned}
n=\frac{2\left(Z_{1-\frac{\alpha}{2}}+Z_{1-\beta}\right)^{2} \sigma^{2}}{\left(\mu_{1}-\mu_{2}\right)^{2}} \\
=\frac{2(1.98+0.84)^{2} 1.48^{2}}{(10.63-9.38)^{2}}=22
\end{aligned}
$$

Therefore, a total of 22 patients referring to Hamadan Dental School and a private office, were recruited. The inclusion criteria consisted of patients requiring extraction of two bicuspids, under the age of 30 years old with no previous orthodontic treatment, with intact enamel, and without attrition, restoration, or caries. The subjects who had craniofacial anomalies, systemic disease, or obvious hypoplasia and demineralization 
were excluded. Each patient was given a manual toothbrush (Oral-B, Procter \& Gamble, CA, USA), a toothpaste containing $1450 \mathrm{ppm}$ (parts per million) fluoride (Colgate, ColgatePalmolive, CA, USA), and similar oral hygiene and diet instructions. A random sequence was generated using random number tables. Right and left quadrants were randomly allocated to control (conventional AE) and experimental (SEP) groups. Allocation concealment was done with opaque numbered sealed envelopes. Blinding of the operator was not possible during bonding but outcome assessment blinding was performed. A total of 44 Roth 0.018-inch stainless steel standard edgewise brackets (Dentaurum, Ispringen, Germany) were bonded to the middle of the buccal surface at least $1 \mathrm{~mm}$ above the gingival margin and were light-cured (Kerr, Orange, CA, USA). Prophylaxis, with slurry pumice and a low-speed handpiece, rinsing, drying, and isolation with cheek retractors, cotton rolls, and saliva ejector were performed for both groups before bonding.

The clinical procedures for bonding in the control group were as follows:

- Etching with $37 \%$ phosphoric acid gel for 15 seconds,

- Thorough rinsing and air-drying to see a frosty appearance,

- Applying a thin layer of Transbond XT resin (3M Unitek, Monrovia, CA, USA) to the bracket base,

- Firmly placing the bracket and removing the excess paste with a scaler,

- Light-curing for 20 seconds $(10$ seconds from the mesial aspect and 10 seconds from the distal aspect of the bracket).

The clinical procedures for bonding in the experimental group were as follows:

- Applying Transbond Plus SEP (3M Unitek, Monrovia, CA, USA) to the enamel surface and rubbing for 3-5 seconds,

- Removing the excess SEP with air-drying for 1-2 seconds,

- Applying a thin layer of Transbond XT resin to the bracket base,

- Firmly placing the bracket and removing the excess paste with a scaler,

- Light-curing for 20 seconds (10 seconds from the mesial aspect and 10 seconds from the distal aspect of the bracket).

The bonded brackets were incorporated into a stainless steel or nickel-titanium (Ni-Ti) wire, depending on the initial crowding, to start the orthodontic treatment. The teeth were maintained in the oral cavity for two months, and thereafter, they were extracted by a periodontist using the Ultrasonic Bone Surgery System (VarioSurg, NSK, Tokyo, Japan) to prevent bracket debonding during extraction (Fig. 1).

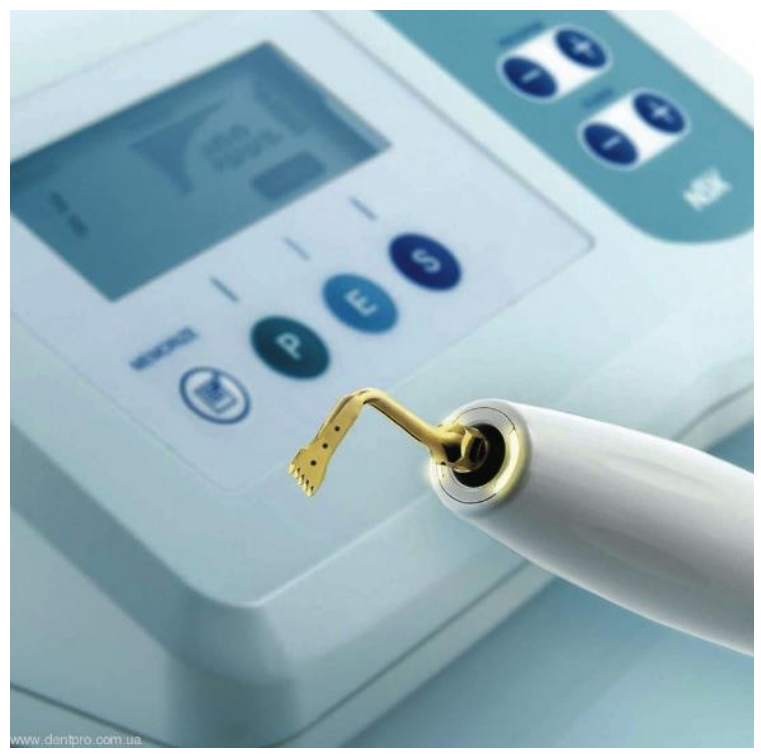

Fig. 1. Ultrasonic Bone Surgery System (VarioSurg, NSK, Tokyo, Japan)

The extracted teeth were embedded in $0.1 \%$ thymol solution (Mylan Seiyaku, Japan) to preclude bacterial growth. They were then mounted in a self-cure acrylic mold (Ivoclar Vivadent, Naturno BZ, Italy) such that the buccal surface of the specimen was perpendicular to the bottom of the mold. A Universal Instron Testing Machine (Zwick/Roell, Ulm, Germany) was employed to apply an occlusogingival shearing force at the bracket-tooth interface at a crosshead speed of $1 \mathrm{~mm} /$ minute. 


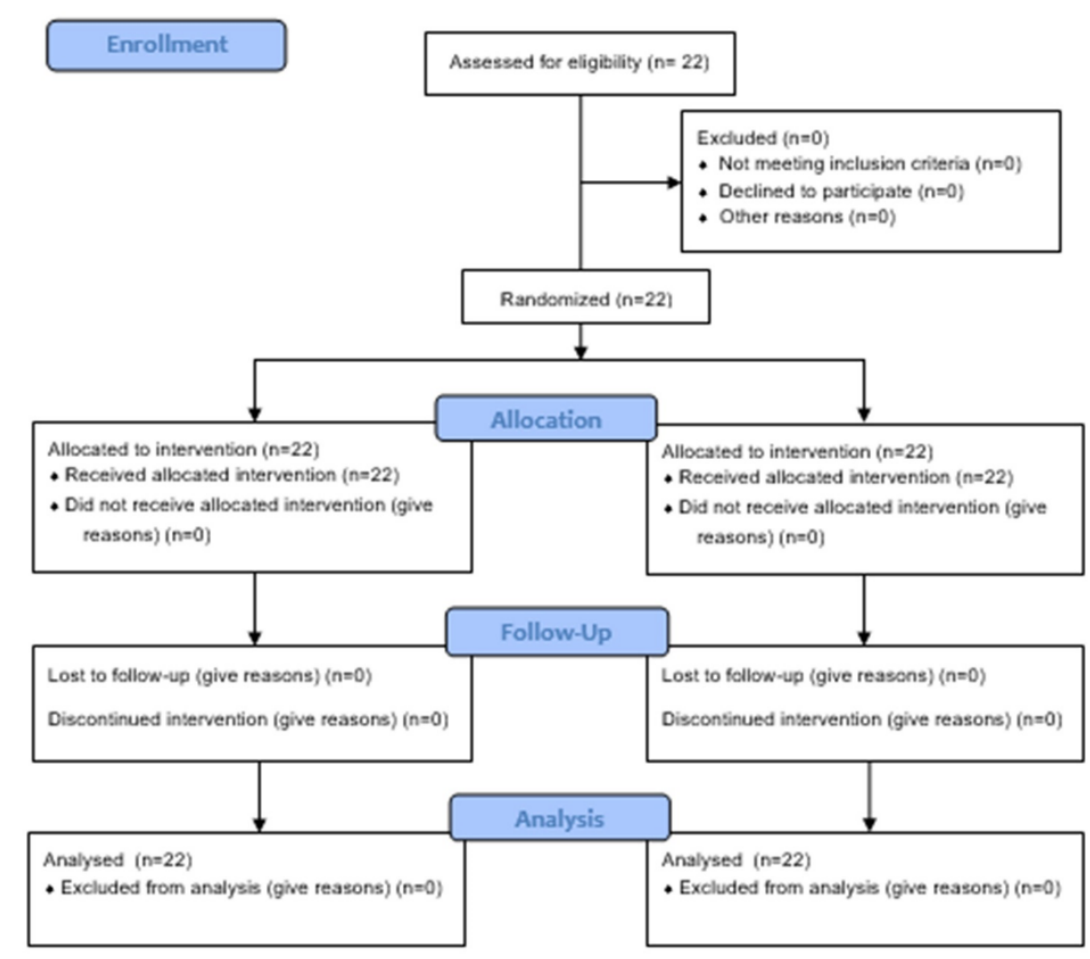

Fig. 2. CONSORT flow diagram demonstrating patient flow through each stage of clinical trial

The force, recorded in Newton $(\mathrm{N})$, was divided by the surface area of the bracket base $\left(16 \mathrm{~mm}^{2}\right)$ to calculate the SBS in Megapascal (MPa). After debonding the brackets, the teeth were assessed under a stereomicroscope (Olympus, SZX16, Guangdong, China) at $\times 5$ magnification to record the adhesive remnant index (ARI). ARI was scored according to the criteria employed by Årtun and Bergland [22] as follows: "score $0=$ no adhesive left on the tooth, score $1=$ less than half of the adhesive left on the tooth, score $2=$ more than half of the adhesive left on the tooth, and score $3=$ all the adhesive left on the tooth, with distinct impression of the bracket mesh". The data were analyzed using SPSS 23 software (IBM Corp., Chicago, IL, USA). Descriptive statistics (mean, standard deviation (SD), the range for SBS, and frequency distribution for ARI scores) were calculated for each group. The normal distribution of data for SBS was checked according to Kolmogorov-Smirnov test.
Due to the paired nature of data, Wilcoxon test was used to analyze the difference in the ARI between the study groups.

\section{RESULTS}

As shown in the CONSORT flow diagram (Fig. 2 ), all the participants (22 subjects; 4 males and 18 females with the age range of 13 to 26 years old and the mean age of 18 years old), who met the inclusion criteria, signed informed consent before being included in the study. No drop-outs were seen in this study. None of the brackets were debonded, neither during the study period nor at the time of extraction. No enamel fracture was observed in either of the groups. Therefore, all 44 brackets were included in statistical analysis. Due to the normal distribution of data, shown by Kolmogorov-Smirnov test $(\mathrm{P}=0.020)$, paired t-test was used to analyze the difference in the SBS between the study groups. The descriptive statistics for SBS is shown in Table 1. 
Table 1. Descriptive statistics for shear bond strength (SBS; MPa) and paired t-test to assess SBS difference between the study groups $(\mathrm{N}=22)$

\begin{tabular}{c|ccc|c} 
Groups & Mean & SD & Range & P-Value* \\
AE & 9.53 & 1.55 & $7.41-12.76$ & \\
SEP & 9.20 & 1.37 & $6.34-11.44$ & 0.096 \\
\hline
\end{tabular}

$\mathrm{AE}=$ conventional acid-etch, $\mathrm{SEP}=$ self-etching primer, $\mathrm{SD}=$ standard deviation

*Paired t-test did not detect any significant differences between the AE and SEP groups

The mean SBS for conventional AE and SEP groups was 9.53 and $9.20 \mathrm{MPa}$, respectively. As shown in Table 1, the paired t-test showed no statistically significant difference between the study groups $(\mathrm{P}=0.096)$. Regarding the ARI score, the most frequent scores seen in conventional AE and SEP groups were 3 and 1, respectively (Table 2).

Table 2. Frequency distribution of adhesive remnant index (ARI) scores and mean ranks of ARI (Wilcoxon test) in the study groups $(\mathrm{N}=22)$

\begin{tabular}{|c|c|c|c|c|c|}
\hline \multirow{2}{*}{ Groups } & \multirow{2}{*}{$\mathbf{0}$} & \multicolumn{3}{|c|}{ ARI Scores* } & \multirow{2}{*}{$\begin{array}{c}\text { Mean } \\
\text { Ranks** }\end{array}$} \\
\hline & & 1 & 2 & 3 & \\
\hline $\mathbf{A E}$ & 0 & 1 & 3 & 18 & 3.50 \\
\hline SEP & 6 & 12 & 2 & 2 & 11.38 \\
\hline
\end{tabular}

$\mathrm{AE}=$ conventional acid-etch, $\mathrm{SEP}=$ self-etching primer ${ }^{*} 0=$ no adhesive remaining on the tooth, $1=$ less than half of the enamel is covered with adhesive, $2=$ more than half of the enamel is covered with adhesive, $3=$ enamel is covered entirely with adhesive

** Wilcoxon test detected significant differences between the groups $(\mathrm{P}<0.001)$.

No ARI score of 0 was seen in conventional AE group. As shown in Table 2, Wilcoxon test revealed a statistically significant difference between the study groups $(\mathrm{P}<0.001)$, indicating a significantly higher amount of residual adhesive on the enamel surface after bond failure in the AE group compared to the SEP group.

\section{DISCUSSION}

This randomized clinical trial (RCT) with a split-mouth design was conducted to measure the SBS of brackets bonded with SEPs compared to conventional $\mathrm{AE}$ technique.
Regarding the SBS, several in-vitro studies have been carried out to reveal the difference between SEPs and conventional AE system. Some authors have suggested that there is no significant difference between the two $[6,14,23]$. On the other hand, some authors have stated that the SBS of orthodontic metal brackets is significantly higher with conventional AE $[17,18,24]$. Others, on the contrary, have demonstrated that the SBS of orthodontic metal brackets is significantly higher in the SEP group $[11,12,20]$.

Pickett et al [25] and Sunna and Rock [5] demonstrated a large difference between clinical and laboratory findings on SBS. They suggested that the results of in-vitro studies should be confirmed with in-vivo studies [5,25]. Pickett et al [25] and Hajrassie and Khier [26] found that in-vivo SBS is significantly lower than in-vitro SBS because of the duration of treatment and the exposure of bonded brackets to acid, saliva, orthodontic forces, masticatory forces, and temperature changes.

The main advantage of the split-mouth design is that the patients act as their control, eliminating the effect of patient compliance and inter-subject variability, which results in smaller sample size and increased study power [27]. According to Pandis et al [27], the most appropriate indication of the split-mouth design is for comparing two types of adhesive as there is no carry-across effect (i.e., not expecting one adhesive to carry across interventional quadrant to the control quadrant) and no period effect (i.e., simultaneous administration of both adhesives).

The present study showed no statistically significant difference between the conventional $\mathrm{AE}$ and SEP groups regarding the SBS (9.53 MPa vs. 9.20 MPa). Although there is no definite minimum value for acceptable SBS in clinical orthodontics, various studies have reported it to be equal to 8-9 MPa [5] and 6-8 $\mathrm{MPa}$ [28]. Cal-Neto et al [29] and Mirzakouchaki et al [21] conducted in-vivo studies to compare the effect of SEPs and conventional AE on SBS; they obtained the same results as ours. They kept the brackets in 
the oral cavity for 30 days before extraction but we doubled this time frame according to their suggestion.

In the conventional $\mathrm{AE}$ group, $\mathrm{ARI}$ score of 3 (full coverage of the enamel surface with adhesive) was the most frequent score, whereas ARI score of 0 (no adhesive on the enamel surface) was not observed at all; this means that bond failure in this group was adhesive failure at the bracket surface. In the SEP group, ARI scores of 0 and 1 (no adhesive and less than half of the adhesive on the enamel surface, respectively) were significantly more frequent compared to the conventional $\mathrm{AE}$ group; this implies adhesive failure at the enamel surface and cohesive failure close to the enamel surface, respectively.

Our study found a statistically significant difference in ARI scores between the two groups $(\mathrm{P}<0.001)$ in accordance with most of the studies that detected less residual adhesive on enamel after bond failure in the SEP group $[6,19,30,31]$. On the contrary, in some studies, bond failure in both groups occurred as an adhesive failure at the bracket surface, and the authors did not report any significant difference in ARI scores between conventional AE and SEP groups [20,21,29].

Conventional AE system takes advantage of mechanical retention in the mesh structure for bonding [7]. Scanning electron microscopic (SEM) studies on the etch pattern of conventional $\mathrm{AE}$ and SEPs show two completely different etch patterns: a distinct honeycombed structure with micro- and macro-tag formation occurs after etching with phosphoric acid, whereas shallower etch pattern with irregular tags without distinct indentation into enamel prisms is evident after SEP application [32]. Minimal etching with SEP indicates that bonding in this system is mainly formed as chemical bonding with calcium in enamel rather than mechanical bonding in a conventional $\mathrm{AE}$ system [33]. It seems that the chemical bond obtained with SEP is higher than the mechanical bond achieved with conventional AE; this can explain why bond failure in the conventional AE group occurs mostly as an adhesive failure at the bracket surface.

Although it has been suggested that more residual adhesive prevents enamel fracture [28], it is clear that less residual adhesive is advantageous for clinicians as it saves more time and reduces the chance of enamel damage during clean-up process [31].

It seems reasonably helpful to design studies evaluating both bond failure rate during the full course of orthodontic treatment and ARI after bracket debonding at the end of orthodontic treatment to achieve a more precise result for clinical use.

\section{CONCLUSION}

The findings of the present study indicated that there is no significant difference in SBS between SEP and conventional AE for bonding orthodontic metal brackets. Also, the amount of residual adhesive on the enamel surface is significantly less with SEP compared to conventional AE. No enamel fracture was observed in either of the groups.

\section{CONFLICT OF INTEREST STATEMENT}

None declared.

\section{REFERENCES}

1. Buonocore MG. A simple method of increasing the adhesion of acrylic filling materials to enamel surfaces. J Dent Res. 1955 Dec;34(6):849-53.

2. Newman GV. Epoxy adhesives for orthodontic attachments: progress report. Am J Orthod. 1965 Dec;51(12):901-12.

3. Zachrisson BJ. A posttreatment evaluation of direct bonding in orthodontics. Am J Orthod. 1977 Feb;71(2):173-89.

4. Kinch AP, Taylor H, Warltier R, Oliver RG, Newcombe RG. A clinical trial comparing the failure rates of directly bonded brackets using etch times of 15 or 60 seconds. Am J Orthod Dentofacial Orthop. 1988 Dec;94(6):476-83.

5. Sunna S, Rock WP. Clinical performance of orthodontic brackets and adhesive systems: a randomized clinical trial. Br J Orthod. 1998 Nov;25(4):283-7.

6. Bishara SE, Gordan VV, VonWald L, Olson ME. Effect of an acidic primer on shear 
bond strength of orthodontic brackets. Am J Orthod Dentofacial Orthop. 1998 Sep;114(3):243-7.

7. Keim RG, Gottlieb EL, Vogels DS 3rd, Vogels PB. 2014 JCO study of orthodontic diagnosis and treatment procedures, Part 1: results and trends. J Clin Orthod. 2014 Oct;48(10):607-30.

8. Cinader D. Chemical processes and performance comparisons of Transbond Plus self-etching primer. Orthod Perspect. 2001;8:5-6.

9. Fleming PS, Johal A, Pandis N. Self-etch primers and conventional acid-etch technique for orthodontic bonding: a systematic review and meta-analysis. Am J Orthod Dentofacial Orthop. 2012 Jul;142(1):83-94.

10. Jaberi Ansari Z, Moezzizadeh M, Valizadeh Haghi H. Effect of Different Methods of Blood Decontamination on Resin-Resin Micro-Shear Bond Strength. Avicenna J Dent Res. 2017;9(3): e60711-e60711.

11. Bishara SE, Oonsombat C, Soliman MM, Warren JJ, Laffoon JF, Ajlouni R. Comparison of bonding time and shear bond strength between a conventional and a new integrated bonding system. Angle Orthod. 2005 Mar; 75(2):237-42.

12. Rajagopal $\mathrm{R}$, Padmanabhan $\mathrm{S}$, Gnanamani J. A comparison of shear bond strength and debonding characteristics of conventional, moisture-insensitive, and selfetching primers in vitro. Angle Orthod. 2004 Apr;74(2):264-8.

13. Arnold RW, Combe EC, Warford JH Jr. Bonding of stainless steel brackets to enamel with a new self-etching primer. Am J Orthod Dentofacial Orthop. 2002 Sep;122(3):274-6.

14. Dorminey JC, Dunn WJ, Taloumis LJ. Shear bond strength of orthodontic brackets bonded with a modified 1-step etchant-andprimer technique. Am J Orthod Dentofacial Orthop. 2003 Oct;124(4):410-3.

15. Nanjannawar LG, Nanjannawar GS. Effects of a self-etching primer and 37\% phosphoric acid etching on enamel: a scanning electron microscopic study. J Contemp Dent Pract. 2012 May;13(3):280-4.

16. Turk T, Elekdag-Turk S, Isci D. Effects of self-etching primer on shear bond strength of orthodontic brackets at different debond times. Angle Orthod. 2007 Jan;77(1):108-12.

17. Aljubouri YD, Millett DT, Gilmour WH. Laboratory evaluation of a self-etching primer for orthodontic bonding. Eur J Orthod. 2003 Aug;25(4):411-5.

18. Grubisa HS, Heo G, Raboud D, Glover KE, Major PW. An evaluation and comparison of orthodontic bracket bond strengths achieved with self-etching primer. Am J Orthod Dentofacial Orthop. 2004 Aug;126(2):213-9.

19. Shaikh MA, Kamat NV, Chalakkal P. Effects of light curing of conventional and selfetching primers on shear bond strength. J Clin Orthod. 2014 Apr;48(4):231-7.

20. Buyukyilmaz T, Usumez S, Karaman A. Effect of self-etching primers on bond strength--are they reliable? Angle Orthod. 2003 Feb;73(1):64-70.

21. Mirzakouchaki B, Shirazi S, Sharghi R, Shirazi S, Moghimi M, Shahrbaf S. Shear bond strength and debonding characteristics of metal and ceramic brackets bonded with conventional acid-etch and self-etch primer systems: An in-vivo study. J Clin Exp Dent. 2016 Feb 1;8(1):e38-43.

22. Artun J, Bergland S. Clinical trials with crystal growth conditioning as an alternative to acid-etch enamel pretreatment. Am J Orthod. 1984 Apr;85(4):333-40.

23. Iijima M, Ito S, Muguruma T, Saito T, Mizoguchi I. Bracket bond strength comparison between new unfilled experimental self-etching primer adhesive and conventional filled adhesives. Angle Orthod. 2010 Nov;80(6):1095-9.

24. Bishara SE, VonWald L, Laffoon JF, Warren JJ. Effect of a self-etch primer/adhesive on the shear bond strength of orthodontic brackets. Am J Orthod Dentofacial Orthop. 2001 Jun;119(6):621-4.

25. Pickett KL, Sadowsky PL, Jacobson A, Lacefield W. Orthodontic in vivo bond strength: comparison with in vitro results. Angle Orthod. 2001 Apr;71(2):141-8.

26. Hajrassie MK, Khier SE. In-vivo and in-vitro comparison of bond strengths of orthodontic brackets bonded to enamel and debonded at various times. Am J Orthod 
Dentofacial Orthop. 2007 Mar;131(3):38490.

27. Pandis N, Walsh T, Polychronopoulou A, Katsaros C, Eliades T. Split-mouth designs in orthodontics: an overview with applications to orthodontic clinical trials. Eur J Orthod. 2013 Dec;35(6):783-9.

28. Reynolds R. A review of direct orthodontic bonding. $\mathrm{Br} \mathrm{J}$ Orthod. 1975;2(3):171-8.

29. Cal-Neto JP, Miguel JA, Zanella E. Effect of a self-etching primer on shear bond strength of adhesive precoated brackets in vivo. Angle Orthod. 2006 Jan;76(1):127-31. 30. Bishara SE, Gordan VV, VonWald L, Jakobsen JR. Shear bond strength of composite, glass ionomer, and acidic primer adhesive systems. Am J Orthod Dentofacial Orthop. 1999 Jan;115(1):24-8.

31. Murfitt PG, Quick AN, Swain MV, Herbison GP. A randomised clinical trial to investigate bond failure rates using a selfetching primer. Eur J Orthod. 2006 Oct;28(5):444-9.

32. Zachrisson BU, Buyukyilmaz T. Bonding in orthodontics. In: Graber LW, Vanarsdall RL, Vig KWL, Huang GJ (editors). Orthodontics: Current Principles and Techniques. St Louis: Elsevier, 2017:813-62.

33. Swartz ML. Comprehensive fixed appliance therapy. In: McNamara JA, Brudon WL (editors). Orthodontics and Dentofacial Orthopedics. Ann. Arbor, MI: Needham Press Inc., 2001:149-151. 